\title{
Escuelas, familias y tramas sociourbanas: entrecruzamientos en contextos de diversidad y desigualdad social
}

\author{
María Rosa Neufeld' \\ Laura Santillán" \\ Laura Cerlettil"
}

I- Universidad de Buenos Aires, Buenos Aires, Argentina.

Contacto: mariarosaneufeld@gmail.com

II- Consejo Nacional de Investigaciones Científicas y Técnicas y Universidad de Buenos Aires, Buenos Aires, Argentina. Contactos: laursantillan@gmail.com; laurabcerletti@yahoo.com.ar

\section{Resumen}

En este artículo se presentan algunos aspectos significativos de las investigaciones histórico-etnográficas realizadas por las autoras en los últimos quince años, en escenarios escolares y barrios de la ciudad de Buenos Aires y su conurbación. El objetivo principal es problematizar los procesos escolares y educativos vinculados a las mayorías populares de la Argentina, tomando tres ejes de análisis interconectados. Primero, se hace referencia a las modalidades que caracterizaban la relación entre familias y escuelas a comienzos del siglo actual (que se habían gestado en las últimas décadas del siglo XX), y a las formas en que las políticas sociales de cada momento atravesaban la vida cotidiana escolar y barrial, así como eran apropiadas y significadas por los docentes, las familias y los académicos. Luego, se presentan las formas hegemónicas de representar la relación entre las familias y las escuelas en la actualidad, que la han constituido como una problemática social, caracterizada por particulares requerimientos y demandas tanto para las escuelas como para las familias. Asimismo, se incorpora la presencia de múltiples prácticas y sentidos desplegados por sus protagonistas en su cotidianeidad, que tensionan esas demandas, y articulan con los procesos políticos de mayor generalidad. Finalmente, se puntualizan estas prácticas y sentidos tomando en cuenta las tramas organizativas locales, mostrando los modos en que los procesos escolares y educativos son llevados adelante por múltiples actores sociopolíticos vinculados a diversas formas de acción colectiva, en donde lo escolar se dirime incluyendo y -a la vez- avanzando más allá de la escuela.

\section{Palabras clave}

Escuelas - Familias - Tramas sociourbanas - Diversidad Desigualdad social. 


\title{
Interconnection between schools, families and social-urban: networks in contexts of diversity and social inequality
}

\author{
María Rosa Neufeld" \\ Laura Santillán" \\ Laura Cerletti"
}

I- Universidad de Buenos Aires, Buenos Aires, Argentina.

\begin{abstract}
This article presents certain meaningful features of the historicethnographic investigations carried out by its authors over the past fifteen years in schools and neighborhoods of the City of Buenos Aires and its suburbs. The article aims mainly to question the educational and schooling processes of popular majorities in Argentina, considering three interconnected lines of analysis. First, we refer to way the relation between families and schools was characterized at the beginning of the present century - a mode originated in the late twentieth century - and the social policies of each moment were experienced in the everyday life of schools and neighborhoods, as well as how they were appropriated and signified by teachers, families and scholars. We then present the current hegemonic representations of the relationship between families and schools. These representations have constituted such relationship as a social problem, characterized by specific requirements and demands for schools and for families. We also include multiple practices and meanings that unfold in everyday life, which put a strain on these demands, and relate to larger political processes. Finally, we focus on these practices and meanings considering local organizational networks, showing the way educational and school processes are carried out by multiple socio-political actors linked to different forms of collective action, in which schooling issues are worked out including and at the same time going beyond school.
\end{abstract}

\section{Keywords}

Schools - families - social-urban contexts - diversity - social inequality. 
En este artículo presentamos algunos aspectos significativos de las investigaciones en las que las tres autoras hemos estado implicadas: nos une el haber sido directora e integrantes de uno de los proyectos iniciales que aún en la actualidad siguen siendo parte del Programa de Antropología y Educación (Instituto de Ciencias Antropológicas, Facultad de Filosofía y Letras, Universidad de Buenos Aires) al que pertenecemos. Vamos a poner el foco en la experiencia de investigación que compartimos en el curso de los últimos quince años, en escenarios escolares y barrios de la ciudad de Buenos Aires y localidades de su conurbación.

El eje en el cual nos centraremos aquí tiene que ver con la problematización de los procesos escolares y educativos vinculados a las mayorías populares de nuestro país, Argentina.

Como antropólogas compartimos una concepción de educación amplia y que busca superar los límites etnocéntricos de la noción de escuela, lo cual implica atender a los diversos modos en que lo educativo rebasa lo escolar, al tiempo que los otros planos: la vida cotidiana familiar y barrial, así como la dimensión urbana, económica y política de ninguna manera quedan ante sus puertas.

Algunas de las cuestiones que permean nuestro relato son muy características de la Argentina: la más significativa, seguramente, es la índole del sistema educativo argentino. Desde fines del siglo XIX, y en el contexto de la organización del Estado nación, éste se estructuró como un poderoso dispositivo, pensado para incorporar, integrar a las oleadas migratorias procedentes del continente europeo, junto con los criollos, indígenas y negros que ya lo poblaban. Tempranamente, el sistema educativo argentino llegó a ser, en América Latina, lo más logrado en términos de expansión masiva de la escolarización, con una consigna emancipadora: público, gratuito y laico. Sin embargo, su concreción empírica en las comunidades, familias y escuelas delineaba un cuadro en el que asomaban diversidades y desigualdades notorias, que contrastaba con la imagen ideologizada y divulgada de la Argentina como un país generoso, receptor de migrantes. Los estudios etnográficos registraron, tanto en los intercambios en situaciones públicas como en los espacios escolares, prácticas etnocéntricas y discriminatorias habituales y naturalizadas, dirigidas tanto hacia los migrantes procedentes de países limítrofes o más lejanos, o a los llegados del interior del propio país (NEUFELD; THISTED, 1999; SINISI, 1999). También las afirmaciones, frecuentes en las escuelas, acerca del desinterés de los sectores populares por la educación, o la convicción acerca de que lo que genera el fracaso escolar de muchos niños de las clases subalternas es la distancia entre la cultura que transmite la escuela y lo que han transmitido (u omitido) las familias. Estas formulaciones formaban parte de lo que Elsie Rockwell y Elena Achilli denominan culturas magisteriales (ROCKWELL, 1996; ACHILLI, 2010) pero integraban también el sentido común compartido y campeaban en los textos de algunos investigadores que nos antecedían en la temática (SAUTU; BABINI, 1996). Estos supuestos se transformaron en problemas de investigación, junto con otras formulaciones, con las que fuimos encontrándonos más adelante, tal como la que plantea que determinadas formas de socialización familiar son condición necesaria para una escolarización exitosa.

El contexto que tomamos en cuenta para el análisis que presentamos aquí, los primeros años del siglo XXI, coincide con el período de mayor profundización de la desocupación y pauperización de los sectores populares en la Argentina, con las repercusiones consiguientes en las vidas de los sujetos, en las instituciones educativas, en las tramas barriales y asociativas. Para referirnos a este escenario -y las dinámicas que asumió en la vida de los sujetos-, tomaremos tres ejes de análisis: tres planos que, al tiempo que diferenciables entre sí, entendemos como profundamente interconectados.

Por un lado, haremos referencia a las modalidades que caracterizaban la relación entre familias y escuelas a comienzos del siglo XXI 
(que se había gestado en las últimas décadas del siglo anterior), y a las formas en que las políticas sociales atravesaban al tiempo que constituian la vida cotidiana escolar y barrial, así como eran apropiadas y significadas por los docentes, las familias y, por qué no, los académicos.

Por otro lado, presentaremos las formas hegemónicas de representar la relación entre las familias y las escuelas en la contemporaneidad, que la han constituido como una problemática social, con particulares requerimientos y demandas tanto para las escuelas como para los adultos con niños a su cargo en el ámbito doméstico. Asimismo, incorporaremos la presencia de múltiples prácticas y sentidos desplegados por sus protagonistas en su cotidianeidad, que tensionan esas demandas, y articulan con los procesos políticos de mayor generalidad.

$\mathrm{Y}$ en tercer término, puntualizaremos estas prácticas y sentidos tomando en cuenta las tramas organizativas locales, mostrando los modos en que los procesos escolares y educativos son llevados adelante por múltiples actores sociopolíticos vinculados a diversas formas de acción colectiva, en donde lo escolar se dirime incluyendo y -a la vez- avanzando más allá de la escuela.

\section{Escuelas, políticas sociales y trama urbana a comienzos del siglo $X X \mid$}

A principios del siglo XXI, en las escuelas públicas de la ciudad de Buenos Aires y del conurbano confluían familias de procedencias disímiles y sus niños ${ }^{1}$, con trabajadores de la educación -docentes, directivos, funcionarios-, de jerarquías y posicionamientos políticos y gremiales diversos. Los aunaba ser parte -tanto unos como otros- de las amplias mayorías que sufrían/ resistían hacía más de una década los costos de la instalación del neoliberalismo. Acercarse a sus

1- En buena medida, en las escuelas de Buenos Aires se mezclaban los niños descendientes de las migraciones rural-urbanas internas de mediados del siglo XX y los de oleadas más recientes, principalmente latinoamericanos. problemáticas exigía estrategias de investigación que se alejaran de la microetnografía del salón de clase o de la investigación sociológica tradicional (PALLMA, 2010; NEUFELD, 2010). En esto, la larga tradición holística de la antropología social era una buena ayuda: aportaba la experiencia desarrollada en el estudio de sociedades de pequeña escala. Las abordaba como totalidades pasibles de ser aprehendidas no sólo por sus características acotadas, sino porque el abordaje teórico-metodológico suponía que en las actividades que se desplegaban cotidianamente era posible reconocer, aunque entreverados, los aspectos que en las sociedades modernas cristalizaban en instituciones puntuales. Entonces, cuando los antropólogos comenzaron a abordar las problemáticas educativas de la propia sociedad, pudieron reconocer las fronteras fluidas entre lo que ocurría en las aulas o en los patios escolares, y la vida cotidiana familiar y barrial. Pero la concepción funcionalista de la primera mitad del siglo XX, preocupada por entender cómo las sociedades sostenían su equilibrio, no permitía conceptualizar la conflictividad de la vida social, menos aún las dinámicas propias de la vida urbana ni las presencias estatales. A partir de la apropiación de las perspectivas del marxismo, especialmente en su variante gramsciana, las diferencias sociales pudieron ser pensadas en términos de poder y desigualdad, y los sistemas simbólicos en términos de dominación (CALDEIRA, 1989). Este vuelco teórico puso el foco en las relaciones de poder, entabladas desde lugares desiguales (de dominio, hegemonía, subordinación, resistencia). En los modos en que sucedía esto en cada caso, era posible reconocer formas culturales locales. A partir de aquí sería posible dar cuenta de la dimensión política de la educación a escala de la vida cotidiana (ROCKWELL, 1987; NEUFELD, 1997). La actuación estatal, las políticas públicas, podían ahora ser analizadas en relación a las prácticas de los sujetos que las llevan adelante o las resisten. Debemos puntualizar aquí que las características de la sociedad argentina a partir de los 90 -marcadas por el despliegue y 
encarnamiento del neoliberalismo-, exigieron actualizar los modos de comprensión de lo que entendíamos por Estado, como para reconocer su presencia en espacios cotidianos en momentos en que se pregonaba su desaparición o expresión mínima o para identificarlo en el accionar de las ONGs. Siguiendo la línea abierta por Michel-Rolph Trouillot,

Aunque unido a un número de aparatos, no todos ellos gubernamentales (...) no es un aparato sino un conjunto de procesos. No está necesariamente limitado por alguna institución, ni hay institución que pueda encapsularlo completamente. En ese nivel, su materialidad reside mucho menos en las instituciones que en el discurrir de los procesos y relaciones de poder (TROUILLOT, 2001, p. 128).

La investigación etnográfica puso de manifiesto la notable heterogeneidad de las escuelas de la ciudad de Buenos Aires y su conurbano, vinculada con las características socio-económicas de los barrios en los que se asentaban, pero generada también por la confluencia de niños de procedencias diversas: mientras en algunas la proporción de hijos de migrantes latinoamericanos (bolivianos, paraguayos, peruanos) era importante, en otros casos su cantidad -o la de coreanos o chinosera pequeña, pero se sobredimensionaba y se generaban problemáticas vinculadas con los modos de habla, los modismos y ritmos, las tipificaciones referidas al color, los olores, la supuesta idiosincrasia de cada grupo nacional o étnico (NEUFELD, 1999; SINISI, 1999). Los docentes percibían la superposición entre las problemáticas de la migración y la pobreza, dado que muchos niños, procedentes de villas, pensiones o casas tomadas eran al mismo tiempo recién llegados con las migraciones internas o de países limítrofes.

El panorama fue complejizándose con una crisis económica creciente: en el 2001 se registraban cifras inéditas de desempleo, precarización del trabajo, cancelación de derechos sociales largamente conquistados (como el trabajo en blanco, las obras sociales o las vacaciones). Una parte considerable de las mayorías se encontraba desocupada u ocupada esporádica e informalmente: los maestros, nuestros interlocutores de entonces, hablaban de lo que se vivía en las escuelas así como en los hogares: los niños pequeños, que se dormían en los pupitres porque la noche anterior habían acompañado a sus padres desocupados a cartonear ${ }^{2}$ por los barrios; los padres, que no prestaban ya atención a las tareas escolares ni acompañaban a los maestros en las reuniones de las asociaciones cooperadoras ni en los festejos.

$\mathrm{Al}$ mismo tiempo, ya hacía más de una década que se visibilizaba una pronunciada xenofobia hacia los inmigrantes de países limítrofes y asiáticos (presente en las relaciones sociales cotidianas, en el discurso de la prensa y asomándose también en las escuelas).

Este contexto con su impronta de desigualdades y diversidades permeaba la vida cotidiana de docentes y niños. Cuando hablamos de contexto, nos referimos al tramo que optamos por analizar de un proceso continuo, de larga duración, el llamado presente historizado (ROCKWELL, 2007; NEUFELD, 1997). No pensamos en contexto como marco ni como lo que bordea a nuestro centro de atención. En este sentido, las escuelas no son islas en barrios estigmatizados: así como la pobre infraestructura urbana no está afuera sino afuera y adentro -la inundabilidad del barrio se manifiesta en el agua de las napas que afloran en el patio escolar- del mismo modo las estigmatizaciones que recaen sobre el barrio o los alumnos avanzan sobre las escuelas (la cercanía de las villas miseria o la abundancia de niños bolivianos, por poner un ejemplo) funcionan como mancha estigmatizante.

La dupla diversidad/desigualdad se expresa de múltiples modos articulando problemáticas

2- Con el nombre de cartoneo se alude a la recolección de material reciclable (plástico, papel, cartones) generalmente recogido en la vía pública, para su posterior venta. 
educativas y trama urbana. Una de las lecciones de la investigación etnográfica es que las relaciones de diversidad adquieren densidad en su articulación con las condiciones puntuales generadas por las relaciones de desigualdad de las sociedades actuales.

A su vez, las desigualdades específicamente educativas están profundamente ligadas a las inequidades propiamente urbanas, dado que el Estado, que fue incorporando a las distintas zonas la infraestructura de servicios, ha ido contribuyendo a estas desigualdades demorando u omitiendo la creación de escuelas en las zonas más rezagadas.

Ya en los 90 los barrios de la ciudad de Buenos Aires y el conurbano, poblados por las clases subalternas, eran espacios habitacionales y de sociabilidad, pero también se habían convertido en ámbitos para la implementación de políticas focalizadas (vinculadas con la vivienda, el trabajo, o alimentarias) ${ }^{3}$. Tal fue la profundidad de la crisis que las características de grupos corresidentes o grupos de consumo, que suelen atribuirse a las familias llegaron a suspenderse, dado que el acto mismo de comer a una misma mesa y bajo un mismo techo se fragmentó, distribuyéndose los miembros de los grupos familiares en los comedores comunitarios, escolares o por grupos etáreos. Dentro de la órbita estatal, las escuelas y centros de salud se poblaron de beneficiarios de los distintos planes que realizaban allí las contraprestaciones típicas de estas políticas. El Plan Jefas y Jefes (antes, el Plan Trabajar y otros $)^{4}$ exigían el cumplimiento de horas de tareas comunitarias, generalmente en la cocina escolar o en tareas de limpieza. Paradojalmente, esto permitía que muchas mujeres, que se esforzaban en realizar estas contraprestaciones

3- De acuerdo con los lineamientos de los organismos internacionales (Banco Mundial) se destinaban a población seleccionada según sus NBI (necesidades básicas insatisfechas) y otros indicadores, y reemplazaban criterios más universalistas de asignación.

4- El Programa Jefas y Jefes de Hogar Desocupados. Derecho Familiar de Inclusión Social se creó a través del Decreto N 565/02 en el año 2002 y postuló entre sus objetivos: propender a la protección integral de la familia y asegurar la concurrencia escolar de los hijos y propiciar, en su caso, la incorporación de los jefes y jefas de hogar desocupados a la educación formal o su participación en cursos de capacitación. en las escuelas a las que asistían sus hijos, pudieran verlos, o ser incluso ellas las que les sirvieran el plato de comida.

Si bien estas políticas salían al encuentro de problemas insoslayables, contribuyeron a actualizar representaciones que venían de lejos, que asociaban pobreza y (falta de) cultura: en la cotidianeidad de las escuelas y los barrios, se ahondó el hiato entre escuelas marginales (en las que era preciso asistir, contener y en las que no era posible enseñar) y las que seguían cumpliendo sus objetivos tradicionales. Esto generó un verdadero ranqueo de escuelas, notable proceso de co-construcción en torno de la tipificación de las escuelas de las peores a las mejores que se desarrollaba entre las familias, el personal de las escuelas y también los niños que asistían a las mismas. Sin embargo, había un generalizado movimiento que expresaba resistencia: no todos parecían resignarse, y no sólo los padres acomodados o de sectores medios, también los pertenecientes a sectores subalternizados urbanos, intentaban evitar las escuelas desprestigiadas buscando inscribirlos en las más consideradas.

En el plano académico, se volvió frecuente la referencia a la educabilidad (NEUFELD; THISTED, 2004), buscando explicar por qué la educación no es igualmente exitosa con todos o incluso es imposible en algunos casos. Un texto de Néstor López y Juan Carlos Tedesco aportaba una definición de educabilidad entendida como "el conjunto de recursos, aptitudes o predisposiciones que hacen posible que un niño o adolescente pueda asistir exitosamente a la escuela" (LÓPEZ; TEDESCO, 2002, p. 7) y señalaban que

[...] educabilidad (...) da cuenta del desarrollo cognitivo básico que se produce en los primeros años de vida -vinculado a una adecuada estimulación afectiva, buena alimentación y salud- y la socialización primaria mediante la cual los niños adquieren los rudimentos de un marco básico que les permite incorporarse a una situación 
especializada distinta de la familia, como lo es la escuela (...) Todo niño es potencialmente educable, pero el contexto social opera, en muchos casos, como obstáculo que impide el desarrollo de esta potencialidad (NEUFELD; THISTED, 2004, p. 86).

La última década, sin embargo, logra una impronta distinta. A partir del período que se inicia en el año 2003, en la Argentina hubo un sensible mejoramiento de los indicadores macroestructurales, al tiempo que diferentes políticas fueron jalonando el reposicionamiento del Estado en el campo de los derechos humanos, de la ampliación de coberturas sociales para la niñez, como la Asignación Universal por Hijo $^{5}$, o vinculadas específicamente con la educación, como el aumento de la inversión educativa desde el presupuesto nacional, o la política nacional de entrega de netbooks para los jóvenes que asisten a escuelas secundarias, distintos modos en que se buscó disminuir la brecha social profundizada entre 1976 y 2003.

\section{De la relación entre las familias y las escuelas como una problemática social actual}

Las investigaciones histórico-etnográficas que llevamos adelante nos permiten observar que, a pesar de la importancia de los procesos de los últimos años -en términos de los esfuerzos realizados por revertir las profundas huellas del período de hegemonía neoliberal señalado (1976-2003)-, persisten algunos presupuestos en torno a la escolarización y las familias. Los discursos que en las últimas décadas han hegemonizado -y aún inciden fuertemente- las concepciones sobre la escolarización y educación infantil tienden a señalar, desde sobreentendidos y valores morales no visibilizados como tales, a las familias de los niños. Entre otras, las consideraciones a

5- La Asignación Universal por Hijo, puesta en vigencia en 2009, destina a desocupados, trabajadores del sector informal, o personas que reciban menos que el salario mínimo, una asignación monetaria por cada hijo menor de 18 años o discapacitado, debiendo éstos asistir a instituciones educativas y cumplir controles de salud. las que aludimos diagnostican la ausencia de las familias, su falta de interés y participación en la escuela y la educación, todas cuestiones vistas, además, como inéditas y parte de una pronunciada crisis -actual- en los modelos parentales. Estas representaciones recaen con más fuerza sobre las mayorías subalternas, quienes han sido históricamente destinatarias de una serie de estigmas que aluden a la perpetuación de pautas tradicionales -en estos sectores- y a la falta de capacidad para educar a los hijos -tal como sucede con el concepto de educabilidad mencionado más arriba.

El análisis etnográfico nos ha permitido desmontar esta serie de presupuestos dominantes que presentan como un orden natural experiencias que en realidad se encuentran históricamente configuradas y se constituyen a través de procesos que incluyen importantes cuotas de conflictividad y desigualdad social.

En los registros realizados con maestros y maestras, relevamos con mucha recurrencia una serie de puntualizaciones sobre cómo las familias deben vincularse con la escuela: participar y acompañar (CERLETTI, 2014), que incluyen una serie renovada de demandas ${ }^{6}$. A los reclamos históricos relativos al cumplimiento del horario escolar y la provisión de útiles y materiales, se sumaron otras actividades -muchas de las cuales otrora eran de incumbencia exclusiva del trabajo magisterial-, tales como la supervisión de tareas en el ámbito doméstico, las acciones de apoyo del aprendizaje (relatar cuentos para estimular la oralidad, realizar juegos donde se ponen en práctica contenidos de matemáticas, etc.), y la asistencia a citas, celebraciones y reuniones en que se convoca a las familias, entre otras (CERLETTI, 2014; SANTILLÁN, 2009).

Estas apelaciones respecto al papel de la familia en la escolarización se ponen en juego en el marco de un conjunto amplio de discursos y producción escrita, muchos de ellos por fuera

6- Esta creciente demanda respecto al papel que debe cumplir la familia en la escolarización de sus niños, según han trabajado otros autores, se viene produciendo también en las últimas décadas en otros países (ver por ejemplo NOGUEIRA, 2011). 
de la escuela. Nos referimos a elaboraciones académicas de corte sociológico -tales como las ya citadas respecto a educabilidad, entre otras-, y de diversos organismos nacionales e internacionales, que tienden a considerar determinadas formas de accionar familiar y ciertos modos particulares de vinculación con las escuelas como condición para que la escolarización infantil sea exitosa (o de calidad). La existencia de esta profusa producción, y la frecuencia y la conflictividad con que se alude a la relación entre "la familia y la escuela" se configura en la contemporaneidad en todo un frente discursivo ${ }^{7}$ (FONSECA; CARDARELLO, 2006) que deriva en la presentación de dicha relación como un problema social (CERLETTI, 2014). Estas indicaciones se sustentan en planteos cortoplacistas, sostenidos sobre una crisis de valores que se vislumbra a nivel global, e invisibilizan y/o deslegitiman la variedad de prácticas locales que documentamos desde nuestro registro etnográfico.

Esta problemática, al igual que cualquier otro problema social, no es natural, sino que conlleva un proceso de construcción. Como toda relación social, no está dada de una vez y para siempre, sino que en determinado momento histórico pueden ubicarse cambios y continuidades, transformaciones y reconfiguraciones. Siguiendo a Bourdieu y Wacquant (1998), ha sido decisorio para nosotras no tomar el problema social como viene dado, porque de esa manera se contribuiría a naturalizar (y ocultar) los procesos a través de los cuales se ha constituido como tal, manteniendo la apariencia socialmente construida, y por lo tanto ratificando la doxa.

Si bien por razones de espacio no podemos ahondar en profundidad en la particularidad de los procesos históricos concretos vinculados a la producción social de esta problemática, diremos que a partir de mediados del siglo

7- En alusión a la articulación de discursos que -en torno a un mismo tema- surgen de la negociación entre diversos grupos de interés y que en buena medida tienden a reificar una serie de preocupaciones y presupuestos asociados al mismo (FONSECA; CARDARELLO, 2006). pasado, se produce en nuestro país una gran divulgación de distintas corrientes provenientes de la psicología, así como también experiencias que derivan de ella -entre las cuales se destaca la propuesta de Escuela para Padres $^{8}$ - que implicaron una redefinición respecto a las visiones sobre la distribución de obligaciones y responsabilidades respecto a la infancia, ampliando a las mayorías populares algunos parámetros que podríamos identificar como propios de los (heterogéneos, vale decirlo) sectores medios. De tal forma, determinadas representaciones y modalidades de acción se han ido construyendo como condición necesaria para la escolarización infantil, dando lugar a esta reconfiguración de las demandas $\mathrm{y}$ formas de ponderar y relacionarse con las familias (CERLETTI, 2013). No sólo se trata de las demandas a las familias en cuanto al acompañamiento en la escolaridad que expusimos antes, sino también que la misma sea capaz de brindar un ambiente adecuado (según las normas y valores del modelo de familia nuclear occidental) a los niños (SANTILLÁN; CERLETTI, 2011).

Pasando de lleno al terreno de las prácticas, estos postulados producen importantes desigualdades entre las distintas clases sociales. Para las mayorías subalternas se abren nuevas interpelaciones, que se suman a los históricos juicios sobre su falta de capacidad para orientar adecuadamente a los hijos en la educación. Por tanto, en relación a bases materiales profundamente desiguales - y no a su falta de capacidad -, amplios conjuntos de padres y familias quedan afuera de la posibilidad de cumplir con muchos de los requerimientos relativos a un mayor acompañamiento en la enseñanza y la realización de las actividades escolares (sobre todo aquellas relativas al afianzamiento de los contenidos curriculares).

8 - En el período mencionado hubo diversas experiencias llamadas Escuela para Padres; sobresalió la llevada adelante por la psicóloga Eva Giberti, que junto al pediatra Florencio Escardó, organizó las Escuela para Padres en el Hospital de Niños (este último era jefe de servicio alli) con recomendaciones sobre la crianza de los hijos. 
A la vez, la fijación de los requisitos que se expresan en este frente discursivo (FONSECA; CARDARELLO, 2006), tiene como peculiaridad la de obscurecer un amplio y diversificado espectro de prácticas, tal como desarrollaremos a continuación, que se ponen en juego en los contextos cotidianos de actuación. A partir del registro de la vida cotidiana, el trabajo de investigación etnográfica nos ha permitido relevar prácticas más intersticiales, menos visibles desde otras lógicas, o desde las instituciones formales, que dan cuenta de la presencia y el involucramiento en la escolarización por parte de los padres y/o tutores de los niños que pertenecen a los sectores subalternos. En este sentido, entendemos que lo cotidiano no constituye una dimensión diferente (o por fuera de) la historia, sino justamente es "el “centro' del acaecer histórico” (HELLER, 1972, p. 42). De este modo, la vida cotidiana está impregnada de contenidos históricos, y su análisis delimita y recupera conjuntos heterogéneos de actividades emprendidos y articulados por sujetos particulares (ROCKWELL, 2009).

En los barrios populares en los que trabajamos en nuestras investigaciones, los familiares de los niños realizan numerosas acciones, sostenidas en el tiempo, en torno a la escuela y la educación: consultas y seguimientos que los padres realizan sobre los rendimientos escolares de los hijos, muchas veces interceptando a las maestras y maestros en espacios no necesariamente contemplados por la escuela (en las veredas que rodean a la escuela, en las plazas y la calle), y una serie de iniciativas acerca de lo escolar y educativo que se ligan - siempre en forma dinámica con expectativas y construcciones ancladas en la condición de clase. Entre otros, nos referimos a las decisiones sobre a cuál escuela enviar a los hijos, o cómo distribuir los magros ingresos - e incluso los beneficios sociales que provienen del Estado - para la compra de los requerimientos de la escuela (útiles escolares, libros de textos), así como también las ayudas en cuanto a las tareas que demanda la escuela utilizando muchas veces recursos provenientes de redes barriales, que exceden a los espacios escolares (CERLETTI, 2014; SANTILLÁN, 2012).

Tomadas en conjunto, estas prácticas se destacan porque rebasan los reglamentos y las regulaciones más formalizadas (aquellas producidas desde la misma escuela o desde otros niveles del sistema educativo), lo cual no va en desmedro de la significatividad que revisten para la experiencia escolar de los niños y las niñas. El registro etnográfico de las prácticas cotidianas nos permite advertir que las interacciones entre las familias y los espacios escolares están delineadas por múltiples formas de participación. Algunas de estas interacciones están reguladas por tanto pautadas y legitimadas - por la escuela (convocatorias generales, citaciones y reuniones), mientras que otras son tramadas intersticialmente por los sujetos (en este caso los padres y familiares de los niños) (CERLETTI, 2014). Tomar en cuenta, por tanto, la existencia de estas diversas modalidades de seguimiento de la escolarización - entre las cuales sólo algunas están legitimadas por la escuela a partir de su regulación - contribuye a desterrar miradas que dicotomizan el debate acerca de la presencia o no de la familia en la escuela, y a desnaturalizar los supuestos hegemonizados por el frente discursivo al que aludimos.

Ahora bien, la difusión de discursos que señalan con insistencia la falta de presencia e interés de las familias - sobre todo de las familias económicamente desfavorecidas - en la escuela, soslaya no sólo las prácticas cotidianas que un conjunto amplio de hombres y mujeres ponen en juego, sino también acciones colectivas más amplias y que involucran de manera plena a las realidades de las mayorías populares en nuestro país y región. En lo que sigue abordaremos este punto que nos permitirá complejizar aún más hondamente las relaciones entre familias y escuelas a partir del análisis de los diversos actores que intermedian y en donde la presencia de lo estatal - como venimos advirtiendo - avanza más allá de su fijeza institucional. 


\section{Escuelas, familias y tramas organizativas en torno a la educación}

Cuando nos trasladamos de lleno a los escenarios más próximos de la vida de los chicos, hay huellas palpables de un sinfín de acciones ligadas a la educación y la escolarización, que rebasan claramente a las instituciones más burocratizadas del Estado. Centros de apoyo escolar ${ }^{9}$, merenderos ${ }^{10}$, espacios comunitarios para los niños, son sólo algunas de las iniciativas locales que se despliegan en las villas $\mathrm{y}$ asentamientos populares. Estas presencias, a cargo de actores externos a los barrios y/o de los mismos pobladores, responden -como veremos- a una heterogénea y diversificada trama de intervenciones sociales y políticas, que no necesariamente son reconocidas -al menos en su naturaleza y sostenimiento en el tiempo- por parte de los espacios formales de educación. Sin embargo, no por ello son menos relevantes para ahondar aún más en uno de los aportes clásicos de la etnografía educativa, que es considerar lo educativo más allá de lo escolar, y, como daremos cuenta, también, lo escolar más allá de la escuela.

Como venimos enunciando, la tradición holística de la antropología nos ha orientado a articular en el análisis dimensiones diversificadas de la vida social. Esta decisión teórica ha implicado entender a lo escolar y educativo como experiencia formativa $\mathrm{y}$ colectiva (THOMPSON, 1992; BERTUCCI; FARIA FILHO; TABORDA, 2010), teniendo lugar a través de procesos de diversa índole que se estructuran en una determinada configuración social (ELIAS, 1996). En la etapa que analizamos, actores diferenciados entre sí, construyeron visiones acerca de lo que significa

9- En referencia al trabajo que realizan organizaciones comunitarias y grupos de vecinos con el fin de ayudar a los niños y las niñas del barrio para completar la tarea que solicita la escuela, juntándolos en domicilios particulares y/o edificaciones de las asociaciones.

10- Se llaman merenderos a los espacios comunitarios que abren organizaciones barriales o vecinos, en donde se ofrece a los chicos, a la salida del horario escolar, una copa de leche. educar a los chicos y las chicas de las clases populares, forjando en ese proceso sentidos que avanzaron más allá de las certificaciones y currículas escolares oficiales.

Nos situamos nuevamente en el espacio sociourbano de la metrópolis de Buenos Aires. Un escenario en el cual, como advertimos, en virtud de la crisis del año 2001 se complejizaron procesos de diversidad y desigualdad que venían teniendo lugar, y revitalizaron los estigmas hacia los barrios y sus pobladores (los otros migrantes, desempleados, cartoneros). Aún así, los grupos familiares -junto con actores externos a los barrios- no quedaron inertes e impulsaron diversas estrategias asociativas en función de resolver los problemas más acuciantes, entre ellas la educación y escolarización de los hijos. Los procesos de conflictividad social y los estallidos populares que tuvieron lugar a inicios del siglo $\mathrm{XXI}^{11}$, revitalizaron formas de acción colectiva como las gestadas por los movimientos sociales que en un mediano plazo atravesaron a los barrios y se concatenaron con diversas modalidades organizativas de los pobladores.

En nuestro país, como anticipamos, distintos movimientos y procesos sociales y políticos han producido que de manera progresiva el barrio se convierta para las mayorías populares en un ámbito central -aunque no por ello exclusivo- en donde acontecen y se dinamizan intervenciones de diverso tipo. Si bien, en nuestra región, el barrio históricamente ha constituido un ámbito altamente significativo para la reproducción cotidiana y la sociabilidad entre las clases populares, las orientaciones territorializadas que asumieron las políticas estatales -sobre todo desde los años 90-, así como también las actuaciones que se gestaron desde el propio campo popular (organizaciones de base, movimientos estudiantiles, los pobladores), reconfiguraron los lugares en donde se reside en ámbitos privilegiados para la

11- Nos referimos a las manifestaciones públicas de protesta que tuvieron su hito durante los días 19 y 20 de diciembre del 2001, pero que incluyeron también iniciativas posteriores a esa fecha tales como asambleas barriales y cortes parciales o totales de carreteras, los llamados piquetes. 
organización de demandas y acceso a diversos derechos y recursos materiales (NEUFELD et al., 2002; SANTILLÁN, 2013).

Entre las iniciativas, los centros de apoyo escolar son paradigmáticos para dar cuenta de algunas respuestas que surgen en los barrios en cuanto a la educación de los chicos. La emergencia de estos centros, ubicados en los sectores más internos de las villas y asentamientos populares, no se restringe al año 2001 sino que se retrotrae a las décadas de los 80 y 90. En su organización confluyen estudiantes universitarios, voluntarios sociales, cuadros de base tercermundistas de la Iglesia Católica. Estos actores, junto con la intervención en otras problemáticas que transitaban los barrios, comienzan a impartir a grupos de niños y niñas ayuda en la resolución de las tareas escolares. Lo hacen, en buena medida, impulsando acciones pedagógicas que buscan explícitamente desafiar los formatos tradicionales de la escuela, recuperando para ello la propuesta de la educación popular. En relación a estas acciones, maestros (titulados y no) de los apoyos escolares se fueron configurando rápidamente en interlocutores claves para muchos padres, los pobladores de los barrios, en relación a aspectos cruciales de las trayectorias educativas de sus hijos (la promoción del año, conflictos por el comportamiento de los niños en la escuela, la orientación de estudios secundarios). Una cuestión clave, también, fue el papel que cumplieron -y aun cumpleninterviniendo y mediando en las relaciones entre las familias y las escuelas (resolviendo conflictos, representando a los padres en las entrevistas y citas escolares). Como venimos adelantando, en relación a las trayectorias escolares de los chicos, no hay solo familias y escuelas, entendidas además hegemónicamente como instituciones aisladas -dos bandos- (ACHILLI, 2010; CERLETTI, 2014); sino, en todo caso, un abanico amplio de actores que median entre estas instituciones. Se trata de una intermediación que -aun en organizaciones proclamadas como autonomistas del Estadoprogresivamente fue asumiendo -no sin tensionesayudas y contribuciones estatales para el sostenimiento de las acciones. Todo un conjunto de cuestiones que nos vuelve sobre este punto de comprender lo estatal más allá de sus formas más evidentes e institucionalizadas de intervención.

Ahora bien, aún cuando la presencia de actores externos a los barrios fue central en la etapa inicial de la creación de los centros de apoyo escolar, no menos importantes resultaron las actuaciones que protagonizaron los propios pobladores y grupos familiares de los niños. En la comprensión de las iniciativas comunitarias no podemos pasar por alto los cursos de acción organizativa y política que hombres y mujeres fueron llevando adelante mientras concretaban las ocupaciones masivas de tierras (conformando los llamados asentamientos informales), así también en la urbanización de las villas y la resolución de diversas problemáticas barriales. Como expusieron otros trabajos, las formas populares de hábitat de las villas y asentamientos han implicado tempranamente estrategias asociativas y modalidades renovadas de participación entre los pobladores (CRAVINO, 1998). Sin dudas, este largo proceso ha dejado concreciones y huellas aun palpables en los barrios. Hoy por hoy, en los asentamientos populares, es posible constatar cómo algunos domicilios particulares ceden parte de su espacio a usos más colectivizados que el estrictamente familiar. En los barrios, progresivamente grupos de pobladores fueron asumiendo un lugar protagónico en la generación de acciones relativas a la escolarización y la educación de los niños. En buena medida, entre quienes encaminan las acciones, un punto insoslayable es la experiencia del trabajo territorial dentro de los barrios (SANTILLÁN, 2013). Se trata de una experiencia forjada no sólo en función de la organización de los asentamientos, sino también a partir de la participación activa que pobladores comienzan a tener -sobre todo mujeres- como referentes en la gestión de políticas estatales compensatorias que se implementan desde los fines de los años 80 hasta la actualidad.

Desde los intersticios de las agendas estatales -aunque fuertemente en relación a 
sus dinámicas- grupos de vecinos hacen propia la resolución de aquello que diagnostican: los problemas de ausentismo y fracaso escolar entre los chicos, las dificultades de muchas familias (sus propios contiguos) de cumplir con los requerimientos de la escuela. En relación a las iniciativas antes analizadas, los vecinos y vecinas imprimen sentidos singulares a su tarea diaria. En las iniciativas encaminadas por los pobladores, lo consuetudinario ocupa un lugar muy importante: esto es, la organización diaria del trabajo con los chicos (la división en grupos y la designación de responsables para las actividades), sigue, en buena medida las orientaciones que imponen las costumbres (THOMPSON, 1992). Al respecto, no sólo la circulación de los niños en el espacio (así como los tiempos pautados de entrada y salida) confrontan con las visiones más asentadas en la escuela (acerca de la disciplina, el cuidado, las demarcaciones de lo que es público y lo que es privado), sino que en el diálogo y consejo que los referentes ofrecen a las familias las formas de sociabilidad y conocimiento anclados en el vecinazgo ocupa un papel relevante (SANTILLÁN, 2013). Esto no quita restar atención a los modos en que los pobladores recuperan otras tradiciones (como las provenientes de la escuela común), a través de importantes sincretismos. Todo un conjunto de cuestiones que van asentando particulares maneras de hacer (CERTEAU, 1996), y que implican, entre otras cuestiones, intermediaciones específicas entre las familias de los niños y la escuela.

En nuestra indagación, no pasaron desapercibidos una serie de desafíos y acciones directas que estos referentes barriales plantean a la escuela. Nos referimos a eventos puntuales, en los cuales los pobladores a cargo de los espacios de apoyo escolar se presentan por pedido de los padres y/o iniciativa propia a plantear determinadas cuestiones a los maestros y directivos. En muchos casos el motor es el largo conocimiento que estos referentes tienen en cuanto a la situación (biográfica y/o escolar) de los chicos y las familias (estar al tanto de las condiciones materiales, de algún problema de salud, otro). Por cierto, en estas audiencias improvisadas (o no) sobresale la capacidad de los referentes barriales para neutralizar algunas decisiones de la escuela, aunque en algunos casos también disuadir de sus posturas a los propios padres. Como ya mencionamos, se trata de presencias e interpelaciones a la escolarización formal, que sobresalen por su intermitencia en la planificación y articulación orgánica, pero que, según entendemos, complejizan las visiones hegemónicas que mencionamos anteriormente sobre la falta de interés y participación de los sectores subalternos en la educación.

\section{Palabras finales}

En este artículo avanzamos en la discusión acerca de los sentidos que asumieron las relaciones entre las escuelas y las mayorías populares en nuestro país, tomando en consideración tramas y escenarios sociales específicos. Como dijimos, en el marco de la extensión temprana de la escolarización obligatoria, las visiones que se tornaron hegemónicas fueron aquellas que insistieron en situar a las familias y comunidades de origen (populares) como no aptas, desinteresadas por la educación. Estas visiones, según vimos, se mantuvieron con importantes continuidades, a la vez que se complejizaron al calor de los procesos políticos, sociales y económicos que tuvieron lugar en las últimas décadas del siglo $\mathrm{XX}$ en nuestro país y en el contexto trasnacional.

Las indagaciones etnográficas que hemos desarrollado nos han permitido reconstruir de modo sostenido las maneras y los sentidos que asumen la educación y las relaciones entre las familias y las escuelas en contextos sociales cambiantes, signados además por las formas específicas en que se ha materializado la diversidad y la desigualdad en nuestro país. En referencia a las últimas décadas, vimos cómo la relación entre la educación formal y las clases populares se ha reconfigurado al calor de un conjunto de visiones que se mantienen de manera férrea en 
el tiempo (entre ellas, las alusiones a la falta de interés y capacidad de las clases subalternas por la educación), a las cuales se han sumado nuevas demandas hacia las familias, demandas que son cotidianamente puestas en entre dicho y desafiadas por los familiares de los niños.

Ahora bien, desde la perspectiva teórica y de las investigaciones que llevamos adelante, hemos advertido que las construcciones de lo escolar y lo educativo, así como las relaciones entre las escuelas y las mayorías populares, de ningún modo implican procesos lineales ni acabados, posibles de predecir desde planteos abstractos.

Los procesos de crisis social que atravesaron a las escuelas entre fines y principios del nuevo milenio, dinamizaron procesos históricos que venían de lejos, generando relaciones en las que se combinaban diversidad y desigualdad. Las políticas estatales de asistencia, lejos de ajustarse a una implementación lineal, dieron nueva letra a estigmatizaciones de vieja data acerca de las poblaciones subalternas.

Asimismo, la indagación que llevamos adelante, sustentada en el interés por la dimensión de la vida cotidiana, polemiza con visiones que entienden a las relaciones entre las escuelas y las familias de las clases populares como dos bandos, como instituciones aisladas, sin conexión con actores y tramas sociales más amplias (locales, regionales). Por contrapartida a ello, en las situaciones que documentamos, la atención al escenario social (barrial y urbano), abonó elementos nodales para este desplazamiento de la mirada de lo escolar y educativo desde los formatos más previsibles a los más intersticiales. Todo un conjunto de cuestiones que, según entendemos, desafían lecturas anticipadas, fijas y lineales acerca de cuáles son o deberían ser los sujetos que intervienen en la educación infantil.

$\mathrm{Al}$ respecto, la perspectiva de análisis relacional e histórica, con orientación hacia la totalidad, nos permitió dar cuenta de los modos en que los encuentros entre las clases subalternas y los procesos escolares (en su concreción más clara bajo la forma de escuela y también más allá de ella) se significan, y a la vez recrean, en el terreno de tramas organizativas locales que incluyen y exceden a los actores y procesos previstos en la educación de las jóvenes generaciones.

\section{Referencias}

ACHILLI, Elena. Escuela, familia y desigualdad social: una antropología en tiempos neoliberales. Rosario: Laborde, 2010.

BERTUCCI, Liane; FARIA FILHO, Luciano; TABORDA, Marcus. Edward Thompson: historia e formação. Belo Horizonte: UFMG, 2010.

BOURDIEU, Pierre ; WACQUANT, Löic. Una duda radical. In: BOURDIEU, Pierre; WACQUANT, Löic. Respuestas: por una antropología reflexiva. México: Grijalbo, 1998. p. 177-184.

CALDEIRA, Teresa Pires do Rio. Antropologia e poder: uma resenha de etnografias americanas recentes. BIB: Boletim Informativo e Bibliográfico de Ciências Sociais, Rio de Janeiro, n. 27, p. 3-50, jan./jun. 1989.

CERLETTI, Laura. Familias y escuelas: tramas de una relación compleja. Buenos Aires: Biblos, 2014.

CERLETTI, Laura. Regulaciones y representaciones sobre la vida familiar: de los '60 al presente: responsabilidad parental, niños, madres y maestras en la "Escuela para Padres". In: JORNADAS DE HISTORIA SOCIAL, 4., 2013, Córdoba. IV jornada... Córdoba: La Falda, 2013.

CERTEAU, Michel de. La invención de lo cotidiano I: artes de hacer. México: Universidad Iberoamericana, 1996.

CRAVINO, Cristina. Los asentamientos del Gran Buenos Aires: reivindicaciones y contradicciones. In: NEUFELD, María Rosa et al. (Comps.). Antropología social y política: hegemonía y poder: el mundo en movimiento. Buenos Aires: Eudeba, 1998. p. $261-284$. 
ELIAS, Norbert. La sociedad cortesana. México: Fondo de Cultura Económica, 1996.

FONSECA, Claudia; CARDARELLO, Andrea. Direitos dos mais e menos humanos. In: FONSECA, Claudia; SCHUCH, Patrice (Orgs.). Políticas de proteção à infância: um olhar antropológico. Porto Alegre: UFRGS, 2006. p. 219-251.

HELLER, Agnes. La estructura de la vida cotidiana. In: HELLER, Agnes. Historia y vida cotidiana: aportación a la sociología socialista. Barcelona: Grijalbo, 1972. p. 39-69.

LÓPEZ, Néstor; TEDESCO, Juan Carlos. Las condiciones de educabilidad de los niños y adolescentes en América Latina. Buenos Aires: IIPE, 2002.

NEUFELD, María Rosa. Acerca de antropología social e historia: una mirada desde la antropología de la educación. Cuadernos del Instituto Nacional de Antropología y Pensamiento Latinoamericano, Buenos Aires, n. 17, p. 145-158, 1997.

NEUFELD, María Rosa. Procesos sociales contemporáneos y el desarrollo de la antropología social y política. In: NEUFELD, María Rosa; NOVARO, Gabriela. Introducción a la antropología social y política: relaciones sociales, desigualdad y poder. Buenos Aires: Facultad de Filosofía y Letras; UBA, 2010. p. 7-46.

NEUFELD, María Rosa et al. Sociabilidad y micropolítica en un barrio bajo planes. In: ANDRENACCI, Luciano (Org.). Cuestión social y política social en el Gran Buenos Aires. Buenos Aires: UNGS, 2002. Documentos del Instituto del Conurbano. p. 61-84.

NEUFELD, María Rosa; THISTED, Jens Ariel. El "crisol de razas" hecho trizas: ciudadanía, exclusión y sufrimiento. In: NEUFELD, María Rosa; THISTED, Jens Ariel (Comps.). "De eso no se habla"...los usos de la diversidad sociocultural en la escuela. Buenos Aires: Eudeba, 1999. p. 23-56.

NEUFELD, María Rosa ; THISTED, Jens Ariel. Investigadores implicados: la investigación educativa en espacios barriales de la Ciudad de Buenos Aires. In: NEUFELD, María Rosa; SINISI, Liliana; THISTED, Jens Ariel (Comps.). Docentes, padres y estudiantes en épocas de transformación social. Buenos Aires: Facultad de Filosofía y Letras; UBA, 2010. p. 17-32.

NEUFELD, María Rosa; THISTED, Jens Ariel. 'Vino viejo en odres nuevos': acerca de educabilidad y resiliencia. Cuadernos de Antropología Social, Buenos Aires, n. 19, p. 83-100, jan./jul. 2004.

NOGUEIRA, Maria Alice. A categoria "família" na pesquisa em sociologia da educação: notas preliminares sobre um processo de desenvolvimento. Revista Inter-legere, Natal, n. 9, p. 156-166, jul./dez. 2011.

PALLMA, Sara. Escuelas en contextos de pobreza urbana: construcción de sentidos en el uso en las investigaciones de las categorías de pobreza, exclusión y marginalidad. In: NEUFELD, María Rosa; SINISI, Liliana; THISTED, Jens Ariel (Comps.). Docentes, padres y estudiantes en épocas de transformación social. Buenos Aires: Facultad de Filosofía y Letras, UBA, 2010. p. 47-62.

ROCKWELL, Elsie. Huellas del pasado en las culturas escolares. Revista de Antropología Social, Madrid, v. 16, p. 175-212, 2007.

ROCKWELL,Elsie.Keystoapropriation:ruralschoolinginMexico.In:LEVINSON,BradleyA.;FOLEY,DouglasE.;HOLLAND,DorothyC.(Eds.).The cultural production of the educated person: critical ethnographies of schooling and local practice. Albany:SUNY, 1996. p. 301-324.

ROCKWELL, Elsie. La experiencia etnográfica: historia y cultura en procesos educativos. Buenos Aires: Paidós, 2009.

ROCKWELL, Elsie. Reflexiones sobre el proceso etnográfico, 1982-1985. Documentos DIE - Departamento de Investigaciones Educativas n.13. México DF: Cinvestav, 1987.

SANTILLÁN, Laura. De responsabilidades y demandas que cambian. Revista Novedades Educativas, Buenos Aires, n. 222, p. 14-17, jun. 2009.

SANTILLÁN, Laura. Entre la ayuda y el "desligamiento": prácticas y regulaciones cotidianas sobre las familias y el cuidado infantil en barrios populares del Gran Buenos Aires. Civitas: Revista de Ciências Sociais, Porto Alegre, v. 13, n. 2, p 326-345, maio/ago. 2013.

SANTILLÁN, Laura. Quiénes educan a los chicos: infancia, trayectorias educativas y desigualdad. Buenos Aires: Biblos, 2012.

SANTILLÁN, Laura; CERLETTI, Laura. Familias y escuelas: repensando la relación desde el campo de la antropología y la educación. 
Boletín de Antropología y Educación, Buenos Aires, v. 2, n. 3, p. 7-16, dic. 2011.

SAUTU, Ruth; EICHELBAUM DE BABINI, Ana María. Los pobres y la escuela: trabajos de investigación. Buenos Aires: La colmena, 1996.

SINISI, Liliana. La relación nosotros-otros en espacios escolares "multiculturales": estigma, estereotipo y racialización. In: NEUFELD, María Rosa; THISTED, Jens Ariel (Comps.). "De eso no se habla"... Ios usos de la diversidad sociocultural en la escuela. Buenos Aires: Eudeba, 1999. p. 189-234.

THOMPSON, Edward. Folklore, antropología e historia social. Entrepasados: Revista de História, Buenos Aires, v. 2, n. 2, p. 63-86, 1992.

TROUILLOT, Michel-Rolph. The anthropology of the state in the age of globalization. Current Anthropology, Chicago, v. 42, n. 1, p. 125-138, 2001.

Recibido en: 15.01.2015.

Aprobado en: 02.03.2015

María Rosa Neufeld es antropóloga social (Facultad de Filosofía y Letras, UBA). En dicha facultad, es profesora titular consulta, directora de la Maestría en Antropología Social y del Programa de Antropología y Educación. Se dedica a la investigación histórico-etnográfica de problemáticas en las que se articulan educación y políticas.

Laura Santillán es antropóloga social (Facultad de Filosofía y Letras, UBA). Es investigadora en el Programa de Antropología y Educación de la Facultad de Filosofía y Letras (UBA) y en el Consejo Nacional de Investigaciones Científicas y Técnicas (CONICET). Es docente de la Facultad de Filosofía y Letras (UBA). Indaga en los procesos políticos y sociales involucrados en la crianza y la educación infantil en sectores populares.

Laura Cerletti es antropóloga social (Facultad de Filosofía y Letras, Universidad de Buenos Aires). Es investigadora en el Programa de Antropología y Educación de la Facultad de Filosofía y Letras (UBA) y en el Consejo Nacional de Investigaciones Científicas y Técnicas (CONICET). Es docente de la Facultad de Filosofía y Letras (UBA). Se dedica a la investigación históricoetnográfica sobre problemáticas socioeducativas. 